SHEP-0518, UW/PT 05-16

\title{
Finite-Volume Effects for Two-Hadron States in Moving Frames
}

\author{
C.h. Kim ${ }^{a}$, C.T. Sachrajda ${ }^{a}$ and Stephen R. Sharpe ${ }^{a, b}$ \\ a School of Physics and Astronomy, Univ. of Southampton, \\ Southampton, SO17 1BJ, UK. \\ ${ }^{b}$ Department of Physics, University of Washington, Seattle, WA-98195-1550, USA .
}

\begin{abstract}
We determine the finite-volume corrections to the spectrum and matrix elements of twohadron states in a moving frame, i.e. one in which the total momentum of the twohadrons is non-zero. The analysis is performed entirely within field theory and the results are accurate up to exponential corrections in the volume. Our results for the spectrum are equivalent to those of Rummukainen and Gottlieb which had been obtained using a relativistic quantum mechanical approach. A technical step in our analysis is a simple derivation of the summation formulae relating the loop summations over the momenta of the two hadrons in finite volume to the corresponding integrals in infinite volume.
\end{abstract}




\section{Introduction}

Lattice simulations are necessarily performed in a finite volume. In the evaluation of many quantities of phenomenological interest, such as hadronic masses or matrix elements with at most a single hadron in the external states, the errors caused by the finiteness of the volume decrease exponentially as the volume is increased [1]. In practice, as long as the size of the lattice is large enough compared to the range of the strong interactions, these errors are numerically negligible. This is not the case, however, when two (or more) hadrons are present in external states. For such states the finite-volume effects decrease more slowly, as powers of the box size, $L$, and need to be understood in order to obtain physical quantities with good precision. The theory of such effects has been fully developed for two particles in their rest frame, i.e. with total momentum $\vec{P}=0$. The spectrum of such states was worked out in refs. [2-4] and the finite volume corrections to the matrix elements were obtained in refs. $[5,6]$.

This paper concerns the extension of these results to two-particle states with total momentum $\vec{P} \neq 0$, which we call a moving frame. A generalisation of the results for the spectrum of such states to a moving frame was proposed some time ago in ref. [7]. This was based upon a relativistic quantum mechanical approach in which the two particles satisfied a Klein-Gordon equation with an interaction potential of finite range. Part of the motivation for our project was an attempt to understand some of the steps in the derivation of ref. [7]. We have therefore revisited the problem using an entirely field theoretic approach. Although our final result looks superficially quite different from that obtained in ref. [7], we find that it is possible to transform our expressions into theirs. Thus we confirm, albeit indirectly, their assumptions and methodology. Our derivation and results are useful for a number of reasons. First, the extension of the results for finite volume corrections to matrix elements are new. Second, we give an alternative and simple derivation of the summation formulae that are needed. And, finally, our derivation of the quantization condition for two-particle states is simple and transparent, and in particular does not require the use of an intermediate quantum mechanical theory.

There are several reasons why it is important to have an extension of the theory of finite volume corrections to a moving frame. Some were discussed in ref. [7]: one obtains additional results for the scattering phase shifts at different values of the centre-of-mass momenta from the same lattice configurations; one can more easily study p-wave decays such as $\rho \rightarrow \pi \pi$; and one avoids the need for vacuum subtractions in s-waves. An additional important motivation is provided by the need to control non-perturbative QCD effects in $K \rightarrow \pi \pi$ decays, and hence, for example, to understand quantitatively phenomena such as the $\Delta I=1 / 2$ rule and the value of $\varepsilon^{\prime} / \varepsilon$. Whilst it is true that the decay amplitudes can in principle be evaluated in the rest-frame, the ability to perform simulations in a moving frame will be very important in controlling systematic uncertainties. For example chiral perturbation theory is an important guide in performing the extrapolation of lattice results to those corresponding to physical $u$ and $d$ quark masses. In this approach there are a number of unknown constants, and results from moving frames provide considerably more data points to allow the determination of these low-energy constants (see, for example, ref. [8]). Furthermore, as noted above in the case of scattering 
amplitudes, the use of a moving frame obviates the need for vacuum subtractions in the isoscalar channel. This is important since such subtractions are under poor statistical control.

Throughout this paper we assume that the fields satisfy periodic boundary conditions in the moving frame (and we take the temporal extent of the lattice to be infinite). There has recently been discussion of the possibility of using anti-periodic [9] or twisted boundary conditions $\left(\psi\left(x_{i}+L\right)=\exp \left(i \theta_{i}\right) \psi\left(x_{i}\right) \quad i=1,2,3\right)$ in lattice simulations [10]. Although we do not consider such boundary conditions explicitly in this paper, the techniques presented below, and in particular the summation formulae, can readily be generalised for the different cases.

We now briefly present our result for the finite-volume corrections to the two-particle spectrum in a moving frame. The example we have in mind is of two pions, and so, for concreteness, we will call the particles "pions" throughout this paper. We note, however, that the results in this paper can readily be generalised to the case of two particles with different masses $\left(m_{1}\right.$ and $m_{2}$ say), as long as they are below the inelastic threshold. An important example is the nucleon-pion system. The quantization condition given below (see eq. (150) for the general case) in terms of the centre-of-mass momentum of each particle, $q^{*}$, remains valid. $q^{*}$ is then related to the total centre-of-mass energy $E^{*}$ by the standard kinematical relation $4 q^{* 2}=E^{* 2}-2\left(m_{1}^{2}+m_{2}^{2}\right)+\left(m_{1}^{2}-m_{2}^{2}\right)^{2} / E^{* 2}$. For the remainder of this paper we limit the discussion to the case when the two hadrons are degenerate $\left(m_{1}=m_{2} \equiv m\right)$ but it should be remembered that the generalisation to non-degenerate particles is straightforward.

The finite-volume cubic box breaks the Euclidean symmetry and hence mixes different partial waves. For $\vec{P} \neq 0$, lattice states which are predominantly s-wave also contain components with $l=2,4, \ldots$ (in contrast to the case with $\vec{P}=0$ where the mixing begins at $l=4$ ). The results presented here were obtained under the assumption that only scattering in the s-wave is important in the energy range of interest (which is a good approximation for $K \rightarrow \pi \pi$ decays). The general results are given in the body of the paper. Let the two-pion state have total momentum $\vec{P}$ and let $E$ be one of the energy eigenvalues in the finite-volume $V=L^{3}$. The total energy in the centre-of-mass frame is denoted by $E^{*}\left(E^{* 2}=E^{2}-P^{2}\right)$, and $q^{*}$ is defined by $E^{* 2}=4\left[q^{* 2}+m^{2}\right]\left(2 q^{*}\right.$ is the magnitude of the relative momentum). We find that the spectrum of two-pion states is determined by the relation

$$
\tan \left[\delta\left(q^{*}\right)\right]=-\tan \left[\phi^{P}\left(q^{*}\right)\right]
$$

where $\delta\left(q^{*}\right)$ is the physical s-wave phase-shift and the function $\phi^{P}\left(q^{*}\right)$ is defined by

$$
\tan \left[\phi^{P}\left(q^{*}\right)\right]=\frac{q^{*}}{4 \pi}\left[c^{P}\left(q^{* 2}\right)\right]^{-1}
$$

with the box size entering through the following regularized sum

$$
c^{P}\left(q^{* 2}\right) \equiv \frac{1}{L^{3}} \sum_{\vec{k}} \frac{\omega_{k}^{*}}{\omega_{k}} \frac{e^{\alpha\left(q^{* 2}-k^{* 2}\right)}}{q^{* 2}-k^{* 2}}-\mathcal{P} \int \frac{d^{3} k^{*}}{(2 \pi)^{3}} \frac{e^{\alpha\left(q^{* 2}-\vec{k}^{* 2}\right)}}{q^{* 2}-\vec{k}^{* 2}} .
$$


The summation in this expression runs over the usual finite-volume momenta for periodic boundary conditions: $\vec{k}=(2 \pi / L) \vec{n}$, with $\vec{n}$ a vector of integers. The corresponding pion energy is $\omega_{k}=\sqrt{\vec{k}^{2}+m^{2}}$. The quantities $\left(\omega_{k}^{*}, \vec{k}^{*}\right)$ are the energy and momentum of the four-vector $\left(\omega_{k}, \vec{k}\right)$ boosted into the centre-of-mass frame. $\mathcal{P}$ indicates that one should take the principal value of the integration and the superscript $P$ recalls that we are considering a two-pion system in a moving frame. The exponential factors in eq. (3) regulate the ultraviolet divergences in the sum and integral, and these divergences cancel in the difference of the two. The remaining $\alpha$ dependence is exponentially suppressed as $L \rightarrow \infty$. Other choices of ultraviolet regulator are of course possible, e.g. the analytic regularization used in refs. $[2,3,7]$. For this presentation we prefer to keep a regulator such as the one in (3), so that the cancellation of UV divergences between the sum and the integral is manifest.

Our result for the quantization condition, eq. (2), differs from that of ref. [7] in the form of the kinematical function. Our $c^{P}\left(q^{* 2}\right)$ of eq. (33) is replaced in ref. [7] by

$$
\frac{1}{\gamma L^{3}} \sum_{\vec{k}} \frac{1}{q^{* 2}-r^{2}}
$$

where $\gamma$ is the Lorentz factor for the transformation between the moving and centre-ofmass frames, and ${ }^{1}$

$$
r^{2}=\frac{1}{\gamma^{2}}\left(k_{\|}-\frac{P}{2}\right)^{2}+\tilde{k}_{\perp}^{2},
$$

with $k_{\|}$and $\tilde{k}_{\perp}$ the components of $\vec{k}$ parallel and perpendicular to $\vec{P}$, respectively. The ultraviolet divergences in eq. (4) are regulated by analytical regularization. In sec. 4 we demonstrate that the expression for $c^{P}\left(q^{* 2}\right)$ in eq. (3) is equal to that in eq. (4)), up to terms which vanish exponentially with the volume. The two quantization conditions are therefore equivalent.

We show in sec. 4 that this equivalence also extends to the general situation in which there are multiple partial waves with non-vanishing phase shifts. In light of this, the discussion given in ref. [7] concerning the group theoretic constraints on the mixing between partial waves, and the numerical values of the kinematical functions remains valid. Thus we do not repeat the discussion in this paper.

In earlier papers $\tan \left(\phi^{P}\right)$ was frequently written in terms of the dimensionless variable $q^{*}(L / 2 \pi)$ (and $m(L / 2 \pi)$ where appropriate). In order to avoid confusion when comparing our results with earlier ones, we underline that our notation is different and the energy and momentum variables in this paper (such as $\vec{k}, \omega_{k}, k^{*}, \omega_{k}^{*}, q^{*}, r$ ) are all dimensionful. ${ }^{2}$ The other principal result of this paper is the expression for the finite-volume corrections in matrix elements of local composite operators between external states, one or both of

\footnotetext{
${ }^{1}$ Ref. [7] uses a slightly different definition of $\vec{r}$ in which the $P / 2$ term in eq. (5) comes with a positive sign. This difference has no effect on the sum in eq. (4), however, since a change of the summation variable $\vec{k} \rightarrow \vec{k}+\vec{P}$ brings our form into that used in Ref. [7]. We find the form in eq. (5) more natural, as will become clear in the subsequent discussion.

${ }^{2}$ Of course this is a matter of choice. We choose to use dimensionful variables because it is the physical energies and momenta which we wish to keep fixed as $L$ is increased.
} 
which contain two hadrons (with an energy below the inelastic threshold). An important application is to $K \rightarrow \pi \pi$ matrix elements. Our results [see eq. (90) below] generalise those of Lellouch and Lüscher [5] and Lin et al. [6] for the centre-of-mass frame.

The issues addressed in this paper have also been considered by Christ, Kim and Yamazaki. Using a related but different approach, they have also confirmed the validity of the results of ref. [7], and derived the generalisation of the result of Lellouch and Lüscher for moving frames. Their paper is being released simultaneously with the present article [11].

The remainder of this paper is organized as follows. In the next section we derive the summation formulae that are needed to evaluate the generic loop summation over the momenta of two-particle states. In sec. 3 we use the summation formulae to derive the quantization condition for two-pion states in finite volume. We then show, in sec. 4. that our results are equivalent to those obtained in ref. [7], and in sec. 5] we verify the quantization condition at lowest non-trivial order in perturbation theory in a $\lambda \phi^{4}$ model of pion interactions. Section [6 contains our derivations of the finite volume corrections to $K \rightarrow \pi \pi$ matrix elements. We present our conclusions in sec. 7

\section{Summation Formulae}

Key ingredients in the calculation of finite-volume effects for two-hadron states are summation formulae relating the sums over the discrete momenta in a finite volume to the corresponding integrals over the continuous spectrum in infinite volume. We start from the Poisson summation formula,

$$
\frac{1}{L^{3}} \sum_{\vec{k}} g(\vec{k})=\int \frac{d^{3} k}{(2 \pi)^{3}} g(\vec{k})+\sum_{\vec{l} \neq \overrightarrow{0}} \int \frac{d^{3} k}{(2 \pi)^{3}} e^{i L \vec{l} \cdot \vec{k}} g(\vec{k}),
$$

where the summation on the left-hand-side is over all integer values of $\vec{n}=\left(n_{1}, n_{2}, n_{3}\right)$, with $\vec{k}=(2 \pi / L) \vec{n}$, while that on the right-hand-side is over integer values of $\vec{l}=\left(l_{1}, l_{2}, l_{3}\right)$ excluding $\vec{l}=(0,0,0)$. In the following, we consider functions $f(\vec{k})$ whose Fourier transforms, $\tilde{f}(\vec{r})$, are non-singular, and are either contained in a finite spatial region or decrease exponentially as $|\vec{r}| \rightarrow \infty$. If we apply the Poisson summation formula to such functions, the terms with $\vec{l} \neq 0$ on the right-hand-side of eq. (6) decrease at least exponentially as the box size is sent to infinity, so that

$$
\frac{1}{L^{3}} \sum_{\vec{k}} f(\vec{k})=\int \frac{d^{3} k}{(2 \pi)^{3}} f(\vec{k})
$$

up to exponentially small corrections. We note that functions $f(\vec{k})$ with these properties have no singularities for real $\vec{k}$, and fall off fast enough at $|\vec{k}| \rightarrow \infty$ that the integrals in eq. (6) converge.

We begin our discussion with a review of the summation formulae in the centre-of-mass frame and then in section 2.2 generalise the discussion to the moving frame. 


\subsection{Summation Formulae in the Centre-of-Mass Frame}

For two-hadron correlators with an energy below the inelastic threshold, the finite-volume corrections are contained in summations of the form ${ }^{3}$

$$
S(\vec{q}) \equiv \frac{1}{L^{3}} \sum_{\vec{k}} \frac{f(\vec{k})}{q^{2}-k^{2}}
$$

where we assume that $q^{2}$ is such that there is no term in the sum with $k^{2} \equiv|\vec{k}|^{2}=q^{2}$ and that $f(\vec{k})$ has the properties discussed above, and in particular has no singularities for real $\vec{k}$. The singularity at $k^{2}=q^{2}$ forbids the simple replacement of the sum with an integral. Defining spherical coordinates, $\vec{k}=(k, \theta, \phi)$, we expand $f$ in spherical harmonics

$$
f(\vec{k})=\sum_{l=0}^{\infty} \sum_{m=-l}^{l} f_{l m}(k) k^{l} Y_{l m}(\theta, \phi)
$$

so that (with $q=|\vec{q}|$ )

$$
S(\vec{q})=\sum_{l, m} S_{l m}(q) \quad \text { where } \quad S_{l m}(q) \equiv \frac{1}{L^{3}} \sum_{\vec{k}} \frac{f_{l m}(k)}{q^{2}-k^{2}} k^{l} Y_{l m}(\theta, \phi) .
$$

In the following, it is convenient to consider each $S_{l m}(q)$ individually.

To make use of eq. (77), we subtract from the summand a function chosen to cancel the pole at $q^{2}=k^{2}$, leading to the result

$$
\begin{aligned}
& \frac{1}{L^{3}} \sum_{\vec{k}} \frac{f_{l m}(k)-f_{l m}(q) e^{\alpha\left(q^{2}-k^{2}\right)}}{q^{2}-k^{2}} k^{l} Y_{l m}(\theta, \phi)= \\
& \int \frac{d^{3} k}{(2 \pi)^{3}} \frac{f_{l m}(k)-f_{l m}(q) e^{\alpha\left(q^{2}-k^{2}\right)}}{q^{2}-k^{2}} k^{l} Y_{l m}(\theta, \phi),
\end{aligned}
$$

which is valid up to terms which are exponentially small in the volume. The exponential factors $\exp \left[\alpha\left(q^{2}-k^{2}\right)\right]$ (with $\left.\alpha>0\right)$ are included so that the subtraction does not introduce ultraviolet divergences. Note that the factor of $k^{l}$ multiplying $Y_{l m}$ is necessary so that the subtraction does not introduce a singularity at $k=0$, and thus invalidate the use of eq. (7).

From eq. (11) we immediately deduce the required summation formulae ${ }^{4}$

$$
S_{l m}(q)=\delta_{l, 0} \mathcal{P} \int \frac{d^{3} k}{(2 \pi)^{3}} \frac{f_{00}(k)}{q^{2}-k^{2}} Y_{00}+f_{l m}(q) \mathcal{Z}_{l m}(q),
$$

\footnotetext{
${ }^{3}$ Since all variables in this subsection correspond to the centre-of-mass frame, we do not introduce the symbol $*$ which we use elsewhere in this paper to distinguish centre-of-mass variables from those in a moving frame.

${ }^{4}$ As above, this equality holds up to exponentially small corrections, but here, and in similar results below, this will not be stated explicitly.
} 
where

$$
\mathcal{Z}_{l m}(q)=\frac{1}{L^{3}} \sum_{\vec{k}} \frac{e^{\alpha\left(q^{2}-k^{2}\right)}}{q^{2}-k^{2}} k^{l} Y_{l m}(\theta, \phi)-\delta_{l, 0} \mathcal{P} \int \frac{d^{3} k}{(2 \pi)^{3}} \frac{e^{\alpha\left(q^{2}-k^{2}\right)}}{q^{2}-k^{2}} Y_{00} .
$$

Here we have used the result that the integral on the right-hand side of eq. (11) vanishes for $l \neq 0$ as the integrand is rotationally invariant. This integral does not vanish, however, for $l=0$, and must be included. Although the integrand in eq. (11) has no pole at $k=q$, in eq. (12) we separate it into two terms each of which does have such a pole. For consistency, the two terms need to be regulated in the same way and the principal value prescription, denoted $\mathcal{P}$, is a natural choice.

The result in eq. (12) was obtained for $l=0$ in ref. [6] [eq. (C.24)] using different methods. Our approach here allows a simple generalisation to all $l$, and, as will be shown in the next subsection, a generalisation to a moving frame. The form of (12) is intuitively reasonable, since, when the summand is singular and thus rapidly varying between grid points, one expects the sum and integral to differ, with the difference proportional to coefficient of the singularity. In terms of the Poisson summation formula, eq. (6) , the presence of the singularity requires one to keep the terms with $\vec{l} \neq 0$.

Our result for the kinematical function $\mathcal{Z}_{l m}(q)$, eq. (13), appears to depend on the parameter $\alpha$. This, however, is misleading: the $\alpha$ dependence only enters in the exponentially small terms which we do not control. ${ }^{5}$ This follows from the derivation itself; the key result, eq. (11), is valid for any $\alpha$, up to exponentially suppressed terms. It can also be seen directly by evaluating the dependence of $\mathcal{Z}_{l m}$ on $\alpha$, which has the advantage of showing the explicit form of the $\alpha$ dependence. For example, using the Poisson summation formula (6), one can show that

$$
\frac{\partial \mathcal{Z}_{00}}{\partial \alpha}=\frac{e^{\alpha q^{2}}}{(4 \pi \alpha)^{3 / 2}} Y_{00} \sum_{\vec{l} \neq 0} \exp \left[-\vec{l} \cdot \vec{l} L^{2} /(4 \alpha)\right]
$$

The general expression for $\partial \mathcal{Z}_{l m} / \partial \alpha$ has the same exponentials multiplied by powers of $L$. This demonstrates that, if $L$ is sent to infinity with $q$ and $\alpha$ fixed in physical units, the result for $\mathcal{Z}_{l m}$ is independent of $\alpha$. In practice, when working at fixed $L$, one should choose $\alpha$ sufficiently small that the $\alpha$-dependent terms are negligible. The simplest choice is to send $\alpha \rightarrow 0^{+}$.

This is one example of the more general result that $\mathcal{Z}_{l m}$ should be independent of the ultraviolet regulator. We have checked this numerically by verifying the equality of our results (in the limit $\alpha \rightarrow 0^{+}$) to those obtained in Refs. [3] using zeta-function regularization. ${ }^{6}$

The summation formulae presented above contain those given in ref. [2]. The latter are given in terms of the sum

$$
\frac{1}{L^{3}} \sum_{\vec{k}}^{\prime} \frac{f(\vec{k})}{q^{2}-k^{2}}
$$

\footnotetext{
${ }^{5}$ Note that for $l=0$ the sum and integral in the definition of $\mathcal{Z}_{00}$ both have an ultraviolet divergence proportional to $\alpha^{-3 / 2}$, but these cancel leaving the exponentially suppressed dependence on $\alpha$.

${ }^{6}$ The precise relation between our $\mathcal{Z}_{l m}$ and the $Z_{l m}$ of refs. $[3,7]$ is given in eq. (74).
} 
with $q^{2}$ chosen to be one of the values of $k^{2}$ and the prime denotes that the terms with $k^{2}=q^{2}$ are removed from the sum. The formulae in ref. [2] can readily be obtained by taking the limit $|\vec{q}| \rightarrow|\vec{K}|$, where $\vec{K}$ is one of the values of $\vec{k}$ appearing in the sum in eq. (8). This is shown explicitly for the case $l=m=0$ in appendix $\mathrm{C}$ of ref. [6].

\subsection{Summation Formulae in a Moving Frame}

We now generalise the discussion to a moving frame, with total energy-momentum $(E, \vec{P})$. As shown below in sec.3. we require the summation formula for sums of the form

$$
S\left(q^{*}\right) \equiv \frac{1}{L^{3}} \sum_{\vec{k}} \frac{\omega_{k}^{*}}{\omega_{k}} \frac{f\left(\vec{k}^{*}\right)}{q^{* 2}-k^{* 2}} .
$$

Note that, while the summation is over the moving frame momenta $\vec{k}=(2 \pi / L) \vec{n}$, with $\vec{n}$ being a vector of integers, it is convenient to rewrite the summand in terms of the centreof-mass momenta $\vec{k}^{*}$ using the Lorentz transformation of eq. (37). It is also convenient, as will be apparent shortly, to pull the Jacobian $\omega_{k}^{*} / \omega_{k}$ out of the function $f\left(\vec{k}^{*}\right)$.

We now proceed as in sec.2.1, writing $\vec{k}^{*}$ is terms of spherical polar coordinates, $\vec{k}^{*}=$ $\left(k^{*}, \theta^{*}, \phi^{*}\right)$, and expanding $f\left(\vec{k}^{*}\right)$ is spherical harmonics

$$
f\left(\vec{k}^{*}\right)=\sum_{l=0}^{\infty} \sum_{m=-l}^{l} f_{l m}\left(k^{*}\right) k^{* l} \sqrt{4 \pi} Y_{l m}\left(\theta^{*}, \phi^{*}\right) .
$$

The factor of $\sqrt{4 \pi}=1 / Y_{00}$ is introduced to simplify the subsequent expressions for $l=0$. Again using eq. (6) we can write down the required summation formula

$$
\begin{aligned}
& \frac{1}{L^{3}} \sum_{\vec{k}} \frac{\omega_{k}^{*}}{\omega_{k}} \frac{f_{l m}\left(k^{*}\right)-f_{l m}\left(q^{*}\right) e^{\alpha\left(q^{* 2}-k^{* 2}\right)}}{q^{* 2}-k^{* 2}} k^{* l} \sqrt{4 \pi} Y_{l m}\left(\theta^{*}, \phi^{*}\right) \\
& \quad=\int \frac{d^{3} k}{(2 \pi)^{3}} \frac{\omega_{k}^{*}}{\omega_{k}} \frac{f_{l m}\left(k^{*}\right)-f_{l m}\left(q^{*}\right) e^{\alpha\left(q^{* 2}-k^{* 2}\right)}}{q^{* 2}-k^{* 2}} k^{* l} \sqrt{4 \pi} Y_{l m}\left(\theta^{*}, \phi^{*}\right) \\
& =\int \frac{d^{3} k^{*}}{(2 \pi)^{3}} \frac{f_{l m}\left(k^{*}\right)-f_{l m}\left(q^{*}\right) e^{\alpha\left(q^{* 2}-k^{* 2}\right)}}{q^{* 2}-k^{* 2}} k^{* l} \sqrt{4 \pi} Y_{l m}\left(\theta^{*}, \phi^{*}\right) .
\end{aligned}
$$

The Jacobian factor $\omega_{k}^{*} / \omega_{k}$ corresponds to the change of integration variables from the laboratory-frame momenta $\vec{k}$ to the centre-of-mass frame momenta $\vec{k}^{*}$. The integral in eq. (19) is the same as that appearing in the derivation of the centre-of-mass summation formulae (apart from the factor of $\sqrt{4 \pi}$ ), i.e. the same as the integral in eq. (11). It is only non-vanishing for $l=0$.

The summation formulae above can be rewritten into a form convenient for subsequent manipulations. For $l=0$ we have

$$
\begin{aligned}
\frac{1}{L^{3}} \sum_{\vec{k}} \frac{\omega_{k}^{*}}{\omega_{k}} \frac{f_{00}\left(k^{*}\right)}{q^{* 2}-k^{* 2}} & =\mathcal{P} \int \frac{d^{3} k^{*}}{(2 \pi)^{3}} \frac{f_{00}\left(k^{*}\right)}{q^{* 2}-k^{* 2}}+f_{00}\left(q^{*}\right) c^{P}\left(q^{* 2}\right) \\
c^{P}\left(q^{* 2}\right) \equiv c_{00}^{P}\left(q^{* 2}\right) & =\frac{1}{L^{3}} \sum_{\vec{k}} \frac{\omega_{k}^{*}}{\omega_{k}} \frac{e^{\alpha\left(q^{* 2}-k^{* 2}\right)}}{q^{* 2}-k^{* 2}}-\mathcal{P} \int \frac{d^{3} k^{*}}{(2 \pi)^{3}} \frac{e^{\alpha\left(q^{* 2}-k^{* 2}\right)}}{q^{* 2}-k^{* 2}},
\end{aligned}
$$


while for $l>0$ the result is

$$
\begin{gathered}
\frac{1}{L^{3}} \sum_{\vec{k}} \frac{\omega_{k}^{*}}{\omega_{k}} \frac{f_{l m}\left(k^{*}\right)}{q^{* 2}-k^{* 2}} k^{* l} \sqrt{4 \pi} Y_{l m}\left(\theta^{*}, \phi^{*}\right)=f_{l m}\left(q^{*}\right) c_{l m}^{P}\left(q^{* 2}\right), \\
c_{l m}^{P}\left(q^{* 2}\right)=\frac{1}{L^{3}} \sum_{\vec{k}} \frac{\omega_{k}^{*}}{\omega_{k}} \frac{e^{\alpha\left(q^{* 2}-k^{* 2}\right)}}{q^{* 2}-k^{* 2}} k^{* l} \sqrt{4 \pi} Y_{l m}\left(\theta^{*}, \phi^{*}\right) .
\end{gathered}
$$

Note that we use $c^{P}$ as a shorthand for $c_{00}^{P}$. As in the centre-of-mass frame, the derivation given above shows that the quantities $c_{l m}^{P}\left(q^{*}\right)$ are independent of $\alpha$ up to exponentially small corrections in the volume. It is straightforward to use the expressions above to evaluate the $c_{l m}^{P}$ numerically.

It is more difficult than in the centre-of-mass frame to use the Poisson summation formula to obtain simple analytic expressions for the $\alpha$ dependence of the $c_{l m}^{P}$, since the terms with $\vec{l} \neq 0$ in eq. (6) involve a Fourier transform with respect to $\vec{k}$ and not $\vec{k}^{*}$. We have studied this issue numerically and have found that the dependence on $\alpha$ for even $l$ is similar to that found in the centre-of-mass frame (see eq. (14)). For odd $l$ on the other hand, the dependence on $\alpha$ appears to be linear, consistent with the presence of terms proportional to $\alpha \exp (-m L)^{7}$. These differences however, do not affect the essential points necessary for control of finite volume effects. We repeat that for fixed $\alpha$ and physical parameters, the $c_{l m}^{P}$ are independent of $\alpha$ up to exponentially small corrections in the volume. In simulations performed at (large) fixed volume, $\alpha$ should be taken to be sufficiently small so as not to enhance numerically the terms which are formally exponentially small in the volume. This can be achieved for example, by taking the limit $\alpha \rightarrow 0+$.

In summary the summation formula for the sum in eq. (16) is

$$
S\left(q^{*}\right)=\mathcal{P} \int \frac{d^{3} k^{*}}{(2 \pi)^{3}} \frac{f\left(\vec{k}^{*}\right)}{q^{* 2}-k^{* 2}}+\sum_{l=0}^{\infty} \sum_{m=-l}^{l} f_{l m}\left(q^{*}\right) c_{l m}^{P}\left(q^{* 2}\right) .
$$

\section{Quantization Condition in Moving Frames}

The two-pion spectrum in finite volume can be determined from the exponential dependence of correlation functions of composite operators. We use

$$
C_{\vec{P}}(t)=\left\langle 0\left|\sigma_{\vec{P}}(t) \sigma^{\dagger}(\overrightarrow{0}, 0)\right| 0\right\rangle
$$

(with time ordering implicit), where $\sigma(\vec{x}, t)$ is an interpolating operator for two-pion states and $\sigma_{\vec{P}}(t)$ is its spatial Fourier transform:

$$
\sigma_{\vec{P}}(t) \equiv \int d^{3} x \sigma(\vec{x}, t) e^{i \vec{P} \cdot \vec{x}}
$$

We will consider the two-pion correlation function in energy space

$$
\widetilde{C}_{\vec{P}}(E)=\int d t e^{-i E t} C_{\vec{P}}(t) .
$$

\footnotetext{
${ }^{7}$ We have checked this in detail for $\vec{P}$ lying along an single lattice axis, and for $l \leq 6$.
} 
In infinite volume $\widetilde{C}_{\vec{P}}(E)$ has a two-pion cut in the $s$-plane (where $s=E^{2}-P^{2}$ is the Mandlestam variable) starting at the branch-point at $s=4 m^{2}$. The quantization of momenta in a finite volume means that the two-pion cut is replaced by a series of poles and the energies corresponding to these poles give the two-pion spectrum. Our aim in this section is to determine the position of these poles. We restrict the energy $E$ to be in the range $0<s<16 \mathrm{~m}^{2}$, so that we can neglect finite volume corrections from four-pion or higher multiplicity intermediate states. The lower limit on $s$ ensures that the energy is positive in all frames.

Although we phrase our discussion in Minkowski space, we note that this same object may be obtained from the Euclidean space correlators calculated in lattice simulations by analytic continuation to imaginary Euclidean energy (which is the approach used in ref. [2]).

The operator $\sigma(\vec{x}, t)$ must be chosen to have overlap with whatever lattice two pion states we are interested in, but the details are not important here. We refer to ref. [7] for discussion of the symmetry group of two pion states for different choices of $\vec{P}$.

For simplicity, and to match the treatment in refs. $[2,7]$, we will take the two pions to be indistinguishable. Although this restricts the scattering to even $l$, we will not display this constraint explicitly. This is because the final formulae can be shown straightforwardly to hold for all $l$ in the case of distinguishable particles, although there are differences by factors of 2 at intermediate stages in the derivation. We will also assume that there is a symmetry, analogous to G-parity for physical pions, which forbids intermediate states with odd numbers of pions. We stress, however, that we make no assumptions about the form of the pion interaction, e.g. we do not rely on chiral perturbation theory or any other expansion scheme. Thus our results are completely general and apply to any two particle system.

It will be helpful below to use the relations between the centre-of-mass variables and those in the moving frame. $E$ and $\vec{P}$ are the total energy and momentum in the moving frame and $E^{*}=\sqrt{E^{2}-P^{2}}=\sqrt{s}$ is the corresponding total centre-of-mass energy. The relative velocity $(\vec{\beta})$ between the centre-of-mass and moving frames is given by

$$
\vec{\beta}=\vec{P} / E
$$

and $E^{*}$ and $E$ are related by

$$
E=\gamma E^{*} \quad \text { where } \quad \gamma=1 / \sqrt{1-\beta^{2}}
$$

It is also useful to recall the definition of $q^{*}$, the magnitude of the relative momentum in the centre-of-mass frame

$$
q^{* 2}=\frac{1}{4} E^{* 2}-m^{2} .
$$

We stress that, in the following, the finite volume summations are always over the movingframe momenta $\vec{k}=(2 \pi / L) \vec{n}$, with $\vec{n}$ a vector of integers.

The correlation function $\widetilde{C}_{\vec{P}}$ can be expressed in terms of the Bethe-Salpeter kernel $K$ through the series shown in fig.1. Since we choose $E$ to lie below the four-pion threshold, 


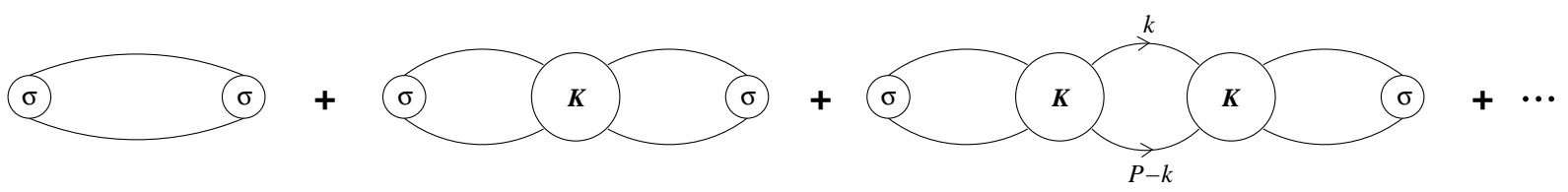

Figure 1: Diagrammatic expansion of the correlator $\widetilde{C}_{\vec{P}}(E)$. Propagators are fully dressed and normalized to unity on shell. $K$ is the amputated two-particle irreducible four-particle correlation function. The circles at the ends represent the operator $\sigma$, renormalized by two factors of $\sqrt{Z}$.

there are no intermediate states with four or more pions and the finite-volume effects in $K$ are exponentially suppressed $[2,6]$. The same is true of the dressed single particle propagators [1]. The only power-law volume corrections arise through the the two pion loops, and we now turn to an analysis of these corrections.

\subsection{Finite Volume Effects in the Generic Loop Summation}

The generic loop integration/summation appearing in fig. 1 is of the form

$$
I \equiv \frac{1}{L^{3}} \sum_{\vec{k}} \int \frac{d k_{0}}{2 \pi} \frac{f\left(k_{0}, \vec{k}\right)}{\left(k^{2}-m^{2}+i \varepsilon\right)\left((P-k)^{2}-m^{2}+i \varepsilon\right)}
$$

where $k=\left(k_{0}, \vec{k}\right)$ and $P=(E, \vec{P})$ are four vectors and we have left out a factor of $i^{2}=-1$ from the propagators which will be accounted for later. The function $f$ contains the energy-momentum dependence arising from the kernels on either "side" of the loop (with one of these kernels replaced by the matrix element of the operator $\sigma$ if the loop lies at one of the "ends" of bubble chains shown in fig. 1) as well as that from the dressed propagators. Since $E$ and $\vec{P}$ are fixed, $f$ can be expressed as a function of the four-vector $\left(k_{0}, \vec{k}\right)$ alone. Note that the dressing of the propagators does not effect the allowed values of the three momenta, which are constrained as usual by the finite volume. The only properties of $f$ that we need are, first, that it has no singularities for real $\vec{k}$ (which holds given our kinematical constraint on $E$ ), and, second, that its ultraviolet behaviour is such as to render the integral and sum convergent.

The power-law volume corrections arise, as usual, because the integrand/summand is singular. To simplify the pole structure we first perform the $k_{0}$ integration. We choose to close the contour of integration so as to pick up the "particle" contribution from the first pole and the "anti-particle" contribution from the second:

$$
I=-i \frac{1}{L^{3}} \sum_{\vec{k}}\left\{\frac{f\left(\omega_{k}, \vec{k}\right)}{2 \omega_{k}\left(\left(E-\omega_{k}\right)^{2}-\omega_{P k}^{2}\right)}+\frac{f\left(E+\omega_{P k}, \vec{k}\right)}{2 \omega_{P k}\left(\left(E+\omega_{P k}\right)^{2}-\omega_{k}^{2}\right)}\right\}
$$

where

$$
\omega_{k}=\sqrt{\vec{k}^{2}+m^{2}} \text { and } \omega_{P k}=\sqrt{(\vec{P}-\vec{k})^{2}+m^{2}} .
$$


We have dropped the factors of $i \varepsilon$ since what remains is a summation, and there is no obstruction to setting $\varepsilon=0$, as long as the poles do not coincide with the allowed values of $\vec{k}$. This will be true in general, since the energies of interest are shifted by interactions away from those of two free particles. This argument does not hold in infinite volume, where we must perform an integral over $\vec{k}$, and the factors of $i \varepsilon$ must be kept. We return to this point below.

For the kinematic region of interest, $0<E^{2}-P^{2}<16 m^{2}$, it is straightforward to show that the only singularity in $I$ is the explicit pole in the first term inside the braces in eq. (32), which occurs at those values of $E$ for which there is a term in the summation with $\omega_{k}+\omega_{P k}=E$. It is this singularity which leads to finite-volume corrections which decrease like powers of the volume. There are no other poles (either explicit or hidden in $f$ ) for $E$ in this kinematical range. For the second term in eq. (32) we can therefore replace the summation by the corresponding integration and write

$$
I=I_{1}+I_{2},
$$

where

$$
\begin{aligned}
& I_{1}=-i \frac{1}{L^{3}} \sum_{\vec{k}} \frac{f\left(\omega_{k}, \vec{k}\right)}{2 \omega_{k}\left(\left(E-\omega_{k}\right)^{2}-\omega_{P k}^{2}\right)} \text { and } \\
& I_{2}=-i \int \frac{d^{3} k}{(2 \pi)^{3}} \frac{f\left(E+\omega_{P k}, \vec{k}\right)}{2 \omega_{P k}\left(\left(E+\omega_{P k}\right)^{2}-\omega_{k}^{2}\right)} .
\end{aligned}
$$

To determine the finite-volume corrections we need to examine $I_{1}$ in more detail. It is convenient to change variables in the summand to those in the centre-of-mass frame. In the moving frame $\vec{k}=\left(k_{\|}, \tilde{k}_{\perp}\right)$ is the momentum of an on-shell particle with mass $m$, where $k_{\|}$and $\tilde{k}_{\perp}$ are the components parallel and perpendicular to $\vec{\beta}$ respectively. The corresponding momentum and energy in the centre-of-mass frame, $\left(k_{\|}^{*}, \tilde{k}_{\perp}^{*}\right)$ and $\omega_{k}^{*}$, are given by

$$
k_{\|}^{*}=\gamma\left(k_{\|}-\beta \omega_{k}\right), \quad \tilde{k}_{\perp}^{*}=\tilde{k}_{\perp} \quad \text { and } \quad \omega_{k}^{*}=\sqrt{k^{* 2}+m^{2}}=\gamma\left(\omega_{k}-\beta k_{\|}\right) .
$$

We now rewrite $I_{1}$ in the form

$$
I_{1}=-i \frac{1}{L^{3}} \frac{1}{E^{*}} \sum_{\vec{k}} \frac{1}{2 \omega_{k}} \frac{f^{*}\left(\vec{k}^{*}\right)}{E^{*}-2 \omega_{k}^{*}}=-i \frac{1}{L^{3}} \frac{1}{2 E^{*}} \sum_{\vec{k}} \frac{\omega_{k}^{*}}{\omega_{k}} \frac{f^{*}\left(\vec{k}^{*}\right)}{q^{* 2}-k^{* 2}} \frac{E^{*}+2 \omega_{k}^{*}}{4 \omega_{k}^{*}},
$$

where $f^{*}$ is the function $f$ rewritten in terms of the centre-of-mass variables. Since $\omega_{k}^{*}$ is a dependent variable, we choose to write $f^{*}$ as a function of $\vec{k}^{*}$ alone.

As expected, we see that the poles correspond to those terms for which $\omega_{k}^{*}=E^{*} / 2$ or equivalently $k^{* 2}=q^{* 2}$. We have deliberately not rewritten the factor $1 / \omega_{k}$ in terms of the centre-of mass variables, so that the summand contains the same Jacobian as in the summation formulae (20) and (22). Using these formulae, we find

$$
I_{1}=-i \frac{1}{2 E^{*}} \mathcal{P} \int \frac{d^{3} k^{*}}{(2 \pi)^{3}} \frac{f^{*}\left(\vec{k}^{*}\right)}{q^{* 2}-k^{* 2}} \frac{E^{*}+2 \omega_{k}^{*}}{4 \omega_{k}^{*}}-\frac{i}{2 E^{*}} \sum_{l=0}^{\infty} \sum_{m=-l}^{l} f_{l m}^{*}\left(q^{*}\right) c_{l m}^{P}\left(q^{* 2}\right),
$$


where we recall that $f_{l m}^{*}$ is defined as in eq. (17) and $c_{l m}^{P}\left(q^{* 2}\right)$ are defined in eqs. (21) and (23).

There is one further step we need to perform in order to write $I_{1}$ as the infinite-volume result together with a correction, i.e. we should replace the principal-value integral in eq. (39) by the corresponding integral with the Feynman $i \varepsilon$ prescription in the propagator and a "delta-function" term:

$$
\begin{aligned}
I_{1}=-i \frac{1}{2 E^{*}} \int \frac{d^{3} k^{*}}{(2 \pi)^{3}} \frac{f^{*}\left(\vec{k}^{*}\right)}{q^{* 2}-k^{* 2}+i \varepsilon} \frac{E^{*}+2 \omega_{k}^{*}}{4 \omega_{k}^{*}} \\
+\frac{q^{*} f_{00}^{*}\left(q^{*}\right)}{8 \pi E^{*}}-\frac{i}{2 E^{*}} \sum_{l=0}^{\infty} \sum_{m=-l}^{l} f_{l m}^{*}\left(q^{*}\right) c_{l m}^{P}\left(q^{* 2}\right) .
\end{aligned}
$$

Note that the "delta-function" term picks out the $l=0$ part of $f^{*}$. Observing that the first term in eq. (40) is exactly the infinite volume expression for $I_{1}$ in Minkowski space (after retracing the steps in the derivation above), we arrive at our final result,

$$
\begin{aligned}
I & =I_{\infty}+I_{F V} \\
I_{F V} & =\left\{\frac{q^{*} f_{00}^{*}\left(q^{*}\right)}{8 \pi E^{*}}-\frac{i}{2 E^{*}} \sum_{l=0}^{\infty} \sum_{m=-l}^{l} f_{l m}^{*}\left(q^{*}\right) c_{l m}^{P}\left(q^{* 2}\right)\right\},
\end{aligned}
$$

where $I_{\infty}$ is the infinite volume result for the original loop integral, $I_{\infty}=I_{1, \infty}+I_{2}$.

The quantity $I_{F V}$ is the desired finite volume correction. Note that it involves the function $f^{*}$ with momentum argument $k^{*}=q^{*}$, so that both particles are on mass shell. Thus the infinite volume amplitudes contained in $f^{*}$ are evaluated with physical momenta, just as if one were picking out the cut in an infinite volume correlator. What is striking about this result is that $q^{*}$ is a momentum determined by the choice of $E$, and can take any positive value. In particular, $q^{*}$ is not constrained by the finiteness of the volume: it does not have to take on one of the (boosted) free particle values. This is a crucial result in the next section where it allows the finite volume corrections to be expressed in terms of physical scattering amplitudes, with the finite volume entering through the purely kinematic functions $c_{l m}^{P}$.

\subsection{Quantization Condition}

In this section we use the result in eqs. (41) and (42) to derive an expression for the twopion energies in finite volume. We first derive the general, though formal, result, next work out the simplest case in which only the s-wave scattering amplitude is non-vanishing, and finally give the result assuming that the scattering amplitude vanishes for $l>l_{\max }$.

Our approach is straightforward: we use eq. (41) for each of the loop summations in fig. 1 . and then reorganize the series. If we keep $I_{\infty}$ in each loop then we recover the infinite volume correlator $\widetilde{C}_{\vec{P}}^{\infty}(E)$. As already discussed, it does not contain the poles which we are seeking. The part of interest is the finite volume correction

$$
\widetilde{C}_{\vec{P}}^{F V}(E)=\widetilde{C}_{\vec{P}}(E)-\widetilde{C}_{\vec{P}}^{\infty}(E)
$$




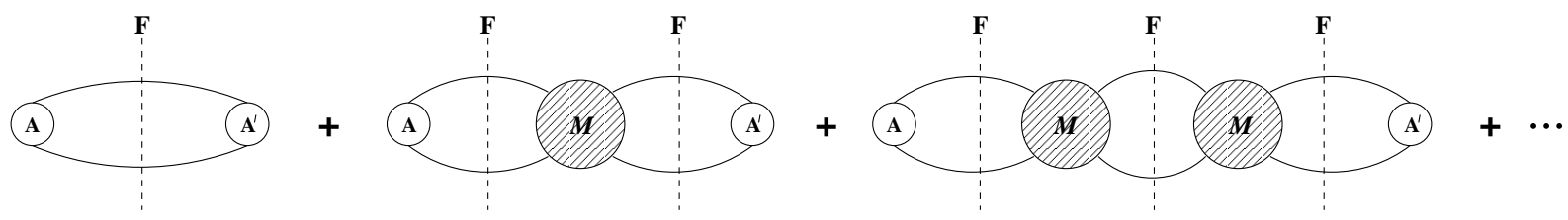

Figure 2: Contributions to the volume dependent part of the $\sigma$ correlator, $\widetilde{C}_{\vec{P}}^{F V}$. The notation is as in fig. 1 except that the filled circles represent the full scattering amplitude, $M$, given by a geometric sum of any number of insertions of the kernel $K$, and the vertical dashed lines indicate that the on-shell finite volume part, $I_{F V}$, has been used for the loop integral. The quantities $A$ and $A^{\prime}$ are defined in the text.

This contains the desired poles, as well as cuts which must cancel those in $\widetilde{C}_{\vec{P}}^{\infty}(E)$. Diagrammatically, $\widetilde{C}_{\vec{P}}^{F V}$ is obtained by keeping at least one insertion of $I_{F V}$. These contributions are shown in fig.2, and lead to the following general result:

$$
\begin{aligned}
\widetilde{C}_{\vec{P}}^{F V}(E) & =-A^{\prime} F A+A^{\prime} F(i M / 2) F A+\ldots \\
& =-A^{\prime} F \frac{1}{1+i M F / 2} A .
\end{aligned}
$$

Here we have taken into account the factor of $i^{2}$ dropped from the loop in the previous section, as well as symmetry factors of $1 / 2$ arising from the identical nature of the particles.

The quantities in eqs. (44) and (45) act on the vector space of states of two pions with total energy $E$ and momentum $\vec{P}$. In particular, $A$ and $A^{\prime}$ are given by

$$
A={ }_{\text {out }}\left\langle\pi \pi ; E, \vec{P}\left|\sigma^{\dagger}(0, \overrightarrow{0})\right| 0\right\rangle Z_{\pi}, \quad A^{\prime}=Z_{\pi}\langle 0|\sigma(0, \overrightarrow{0})| \pi \pi ; E, \vec{P}\rangle_{\text {in }},
$$

and involve a geometric series of contributions with any number of insertions of the kernel $K$, with the intermediate loops containing the infinite-volume expression $I_{\infty}$. The factor of $i \varepsilon$ in $I_{\infty}$ leads to the presence of in- and out-states as shown. $M$ is the on-shell scattering amplitude, obtained by summing a similar geometric series. And, finally, $F$ is a kinematic factor determined by the form of $I_{F V}$, which, in particular, accounts for the fact that the "cut" can have non-vanishing angular momentum.

To give these quantities a concrete representation we change to centre-of-mass variables, since we can then use the partial wave basis. In particular, $M$ is diagonal in this basis, with matrix elements

$$
M_{l_{1}, m_{1} ; l_{2}, m_{2}}=\delta_{l_{1} l_{2}} \delta_{m_{1} m_{2}} \frac{16 \pi E^{*}}{q^{*}} \frac{\left(\exp \left[2 i \delta_{l_{1}}\left(q^{*}\right)\right]-1\right)}{2 i},
$$

where $\delta_{l}$ is the phase shift in the $l$-th partial wave. For $I_{F V}$ we have already changed to centre-of-mass variables in the result (42), from which it follows, using the definition of $f_{l m}^{*}[$ see eq. (17)], and the completeness of the spherical harmonics, that

$$
\begin{aligned}
& F_{l_{1}, m_{1} ; l_{2}, m_{2}}=\frac{q^{*}}{8 \pi E^{*}}\left(\delta_{l_{1} l_{2}} \delta_{m_{1} m_{2}}+i F_{l_{1}, m_{1} ; l_{2}, m_{2}}^{F V}\right) \\
& F_{l_{1}, m_{1} ; l_{2}, m_{2}}^{F V}=-\frac{4 \pi}{q^{*}} \sum_{l=0}^{\infty} \sum_{m=-l}^{l} \frac{\sqrt{4 \pi}}{q^{* l}} c_{l m}^{P}\left(q^{* 2}\right) \int d \Omega^{*} Y_{l_{1}, m_{1}}^{*} Y_{l, m}^{*} Y_{l_{2}, m_{2}},
\end{aligned}
$$


where the implicit arguments of the spherical harmonics are $\left(\theta^{*}, \phi^{*}\right)$, and the asterixes on the spherical harmonics indicate complex conjugation. The integral can be expressed in terms of Wigner 3-j symbols, but an explicit expression will not be needed.

As for the unknown matrix elements $A$ and $A^{\prime}$, the change of variables leads to no simplification, but no complication either. They are simply unknown vectors $A_{l m}$ and $A_{l m}^{\prime}$. Note that, unlike $M$, the matrix elements $A$ and $A^{\prime}$ need not be Lorentz invariant, since the operator $\sigma$ can involve spatially separated pion fields. But this lack of invariance is of no consequence for our argument, since the role of $A$ and $A^{\prime}$ is simply to provide the coupling of the external operators to the on-shell two pion states. In other words, we are interested here in the positions of the poles, and not their residues.

The positions of the poles in $\widetilde{C}_{\vec{P}}^{F V}$ are determined by the factor which lies between $A^{\prime}$ and $A$, namely $F(1+i M F / 2)^{-1}$. $F$ itself inherits from $c_{l m}^{P}$ poles at all energies corresponding to two free pions both having allowed momenta in the moving frame, as is clear from eqs. (21, 23). Turning on the interaction will, however, shift these poles, and indeed one can see that they cancel between numerator and denominator if $M \neq 0$. The poles are shifted to the positions determined by requirement that $1+i M F / 2$ have zero eigenvalue, or equivalently by the formal quantization condition:

$$
\operatorname{det}(1+i M F / 2)=0
$$

This is the main result of this section. It demonstrates that, for any $\vec{P}$, the finite volume energy shifts depend on interactions only through the infinite volume scattering amplitude, as was shown by Lüscher in ref. [3] for $\vec{P}=0$. The result (50) also gives a clear separation between the dynamical effects (the scattering amplitude $M$, independent of the volume) and the kinematical effects of finite volume (the matrix $F$, dependent on the volume, but independent of the scattering amplitude).

In the derivation of ref. [3] it was important that the range of the effective non-relativistic potential, $R$, satisfy $L / 2>R$, aside from an exponentially falling tail. Without this condition there is no "outside" region where the wavefunction is composed of free waves with phase determined by the phase shift. Thus for $L / 2 \leq R$ one would not expect the energy shift to depend only on the phase shifts, i.e. on $M$ alone. By contrast, our result, eq. (50), appears to imply that the energy shifts depend only on $M$ without any proviso. This is, however, misleading. Our entire approach relies on the assumption that sums of non-singular functions in momentum space can be well approximated by integrals. When the range of the potential is larger than the box size, the spread of its Fourier transform $(\Delta k \sim 1 / L)$ is smaller than the spacing between the discrete finite volume momenta. Consequently, the starting assumption of our derivation fails, and there is no conflict with the result of ref. [3].

To make the result (50) into a practical tool we need, following refs. [3,6,7], to truncate the number of partial waves that contribute. In other words, we assume that $\delta_{l}=0$ for $l>l_{\max }$. In the following subsection we discuss the simplest case, $l_{\max }=0$, and then return to the general case where $0<l_{\max }<\infty$. 


\subsubsection{Quantization Condition: s-wave Interactions}

We assume that $A$ and $A^{\prime}$ couple the external operators to two pions with an s-wave component, and that only the s-wave part of the scattering amplitude is non-vanishing. Note that although $F$ couples the s-wave to other waves [through the $c_{l m}^{P}$ terms with $l \neq 0$ in $F^{F V}$, eq. (49)], these couplings would have to contribute through terms of the form $F M F, F M F M F$, etc. in eq. (44) but these vanish since $M=0$ for the higher waves. Thus the geometric series leading to the pole occurs only in the s-wave, and can be treated in isolation. We discuss this point more thoroughly in the general context of the next subsection.

Since the problem is now one-dimensional, the quantization condition is simply

$$
1+i M_{s} F_{s} / 2=0
$$

where

$$
M_{s}=\frac{16 \pi E^{*}}{q^{*}} \frac{\left(e^{2 i \delta\left(q^{*}\right)}-1\right)}{2 i},
$$

with $\delta$ the s-wave phase shift, and $F_{s}$ is the $l=0$ component of $F$ :

$$
F_{s} \equiv F_{00 ; 00}=\frac{q^{*}}{8 \pi E^{*}}-\frac{i}{2 E^{*}} c^{P}\left(q^{* 2}\right) .
$$

We note that the quantization condition (51) has been obtained in ref. [12] using a similar method to ours in the context of a non-relativistic effective theory of nucleon-nucleon scattering. After rearrangement, the quantization condition can be written in the form

$$
\tan \left[\delta\left(q^{*}\right)\right]=-\tan \left[\phi^{P}\left(q^{*}\right)\right] \quad \text { where } \tan \left[\phi^{P}\left(q^{*}\right)\right]=\frac{q^{*}}{4 \pi}\left[c^{P}\left(q^{* 2}\right)\right]^{-1} .
$$

Thus the finite-volume two-pion states on a cubic lattice of size $L^{3}$ are those with energies (and hence values of $q^{*}$ ) which satisfy eq. (54). For the particular case of $\vec{P}=0$ one immediately recovers the Lüscher condition [3] .

We note in passing that for external operators in the moving frame having no centre-ofmass s-wave component, the allowed finite volume energies are those of two free particles.

\subsubsection{Quantization Condition: general interactions}

In this subsection we assume that the phase shifts, and consequently scattering amplitudes, are small enough that they can be ignored for $l>l_{\max }$. In other words, we assume that

$$
M_{l, m ; l, m}=0 \quad \text { if } \quad l>l_{\max } .
$$

As we now explain, this turns out to imply that one can truncate the entire problem to the subspace with $l \leq l_{\max }$. In particular the determinant of the full matrix $(1+$ $i M F / 2)_{l_{1}, m_{1} ; l_{2}, m_{2}}$ vanishes if the determinant of the block with $l_{1}, l_{2} \leq l_{\max }$ vanishes. Thus the quantization condition becomes

$$
\left.\operatorname{det}(1+i M F / 2)\right|_{l_{1}, l_{2} \leq l_{\max }}=0 .
$$


The condition (51) of the previous section is the simplest example $\left(l_{\max }=0\right)$ of this general result.

The subtlety in establishing this result arises from the fact that $F$ has non-vanishing elements connecting $l_{1} \leq l_{\max }$ and $l_{2}>l_{\max }$ and vice-versa. To see that these "block-offdiagonal" matrix elements are irrelevant, it is useful to introduce a projection operator, $P$, onto the subspace with $l \leq l_{\max }$. Then the assumed form of the scattering amplitude, eq. (55), can be written as $M=P M P$. Using this property, and denoting the truncation of $F$ to the $l \leq l_{\max }$ block by $F_{P} \equiv P F P$, one can manipulate the result for the finitevolume part of the correlation function into the form

$$
\widetilde{C}_{\vec{P}}^{F V}(E)=-A^{\prime}\left[F-(i / 2) F M \frac{1}{P+i M F_{P} / 2} F\right] A .
$$

This shows that poles occur when the truncated matrix $P+i M F_{P} / 2$ has zero eigenvalues, which is equivalent to the condition (56).

A consequence of this argument is that we only need to determine $F$ in the truncated basis. Using the explicit form in eqs.(48) and (49), we see that if $l_{1}, l_{2} \leq l_{\max }$, then the angular integral in $F^{F V}$ vanishes for $l>l_{1}+l_{2}$. Thus only a finite number of the quantities $c_{l m}^{P}$ are needed to calculate the truncated determinant.

In order to compare our result to that of refs. $[3,7]$, it is convenient to pull out the volume-independent first term from $F$ in eq. (48). Then one can rewrite the quantization condition as

$$
\left.\operatorname{det}\left(\cos \delta-\sin \delta F^{F V}\right)\right|_{l_{1}, l_{2} \leq l_{\max }}=0
$$

where $\cos \delta$ is a diagonal matrix with entries $\cos \delta_{l_{1}}$ on the diagonal, and similarly for $\sin \delta$. This condition has the same form as eq. (100) of ref. [7], and agrees if our matrix $F^{F V}$ equals that denoted $\mathcal{M}$ there [and by $\mathcal{M}^{R G}$ in eq. (59) below]. We have checked, using the relation between our $c_{l m}^{P}$ and the zeta-functions $Z_{l m}^{d}$ of ref. [7] given in eq. (74) below, that in fact,

$$
F_{l_{1}, m_{1} ; l_{2}, m_{2}}^{F V}=i^{-l_{1}} \mathcal{M}_{l_{1}, m_{1} ; l_{2}, m_{2}}^{R G} i^{l_{2}} .
$$

The extra factors of $i$ cancel in the determinant in eq. (58), because $\cos \delta$ and $\sin \delta$ are diagonal matrices. Thus our quantization condition agrees with that of ref. [7].

\section{Comparison of Quantization Condition with that of Rummukainen and Gottlieb}

In this section we show that the kinematical functions $c_{l m}^{P}\left(q^{* 2}\right)$, defined in eqs. (21) and (23), can be rewritten in the form given in ref. [7]. As already noted in the previous section, this implies the equivalence of the quantization conditions. To compare the results requires a small kinematical exercise, i.e. the rewriting of the factor $\left(\omega_{k}^{*} / \omega_{k}\right) /\left(q^{*^{2}}-k^{*^{2}}\right)$, which appears in the definition of $c_{l m}^{P}\left(q^{* 2}\right)$ in terms of the momentum $r$ of eq. (15). We need this in the vicinity of the pole, i.e. for $q^{* 2} \approx k^{* 2}$. We first recall that, for a given choice of $\vec{k}$, 
setting $q^{* 2}=k^{* 2}$ corresponds to a kinematical situation with both pions on-shell. This follows from the derivation in the previous section, in which the pole in $q^{*}$ arises when both intermediate propagators are on shell. The particular consequence we need here is that $\omega_{k}^{*}=E^{*} / 2$ at the pole, since the two on-shell particles share the energy equally in the CM frame. This result can be seen directly from

$$
\omega_{k}^{* 2}-\left(E^{*} / 2\right)^{2} \equiv\left(k^{* 2}+m^{2}\right)-\left(q^{* 2}+m^{2}\right)=k^{* 2}-q^{* 2} .
$$

An important corollary is that $\vec{r}=\vec{k}^{*}$ at the pole. To see this we recall from ref. [7] that

$$
r_{\|} \equiv \gamma^{-1}\left(k_{\|}-P / 2\right), \quad \tilde{r}_{\perp} \equiv \tilde{k}_{\perp}=\tilde{k}_{\perp}^{*} .
$$

Using the inverse Lorentz transform, $k_{\|}=\gamma\left(k_{\|}^{*}+\beta \omega_{k}^{*}\right)$, together with $E^{*}=E / \gamma$ and $\beta=P / E$, one finds

$$
r_{\|}=k_{\|}^{*}+\beta \omega_{k}^{*}-P /(2 \gamma)=k_{\|}^{*}+\beta\left(\omega_{k}^{*}-E^{*} / 2\right) .
$$

Since $\omega_{k}^{*}=E^{*} / 2$ at the pole, this establishes the desired result. Thus setting $q^{* 2}=k^{* 2}$ implies also $q^{* 2}=r^{2}$, and it follows that the positions of the poles in $q^{*}$ in the sum contained in $c^{P}$, eq. (21), are the same as those in the sum of ref. [7], eq. (41). This is clearly a necessary condition for the equivalence of the results.

It is now straightforward to determine the residue of the pole when written in terms of $r^{2}$ rather than $k^{* 2}$. Working to linear order in the difference $E^{*} / 2-\omega_{k}^{*}$ we have

$$
E^{*} / 2-\omega_{k}^{*} \approx \frac{E^{* 2} / 4-\omega_{k}^{* 2}}{2 \omega_{k}^{*}}=\frac{q^{* 2}-k^{* 2}}{2 \omega_{k}^{*}} .
$$

Using this result in eq. (62) yields

$$
k^{* 2}-r^{2} \approx 2 \beta k_{\|}^{*}\left(E^{*} / 2-\omega_{k}^{*}\right) \approx \beta k_{\|}^{*}\left(q^{* 2}-k^{* 2}\right) / \omega_{k}^{*} .
$$

Thus we find

$$
\begin{aligned}
q^{* 2}-r^{2} & =\left(q^{* 2}-k^{* 2}\right)\left(1+\beta k_{\|}^{*} / \omega_{k}^{*}\right)+O\left[\left(q^{* 2}-k^{* 2}\right)^{2}\right] \\
& =\left(q^{* 2}-k^{* 2}\right)\left(\omega_{k} / \gamma \omega_{k}^{*}\right)+O\left[\left(q^{* 2}-k^{* 2}\right)^{2}\right]
\end{aligned}
$$

where in the second step we have used $\omega_{k}=\gamma\left(\omega_{k}^{*}+\beta k_{\|}^{*}\right)$.

Using this result we can rewrite our kinematical functions in terms of $r^{2}$. We begin with $c^{P}\left(q^{* 2}\right)$ :

$$
\begin{aligned}
c^{P}\left(q^{* 2}\right) & =\frac{1}{L^{3}} \sum_{\vec{k}} \frac{\omega_{k}^{*}}{\omega_{k}} \frac{e^{\alpha\left(q^{* 2}-k^{* 2}\right)}}{q^{* 2}-k^{* 2}}-\mathcal{P} \int \frac{d^{3} k}{(2 \pi)^{3}} \frac{\omega_{k}^{*}}{\omega_{k}} \frac{e^{\alpha\left(q^{* 2}-k^{* 2}\right)}}{q^{* 2}-k^{* 2}} \\
& =\frac{1}{\gamma L^{3}} \sum_{\vec{k}}\left\{\frac{e^{\alpha\left(q^{* 2}-r^{2}\right)}}{q^{* 2}-r^{2}}+g(\vec{k})\right\}-\frac{1}{\gamma} \mathcal{P} \int \frac{d^{3} k}{(2 \pi)^{3}}\left\{\frac{e^{\alpha\left(q^{* 2}-r^{2}\right)}}{q^{* 2}-r^{2}}+g(\vec{k})\right\} .
\end{aligned}
$$

Here $g$ is a non-singular function of $\vec{k}$, which arises from the $O\left[\left(q^{* 2}-k^{* 2}\right)^{2}\right]$ terms in eq. (66), with contributions both from the pole and the exponential. It has an implicit 
dependence on $q^{*}$. Its form is not required, however, since the sum and integral over $g(\vec{k})$ cancel (up to exponentially small terms in the volume) as a consequence of the Poisson summation formula. Thus we find that

$$
c^{P}\left(q^{* 2}\right)=\frac{1}{\gamma L^{3}} \sum_{\vec{k}} \frac{e^{\alpha\left(q^{* 2}-r^{2}\right)}}{q^{* 2}-r^{2}}-\frac{1}{\gamma} \mathcal{P} \int \frac{d^{3} k}{(2 \pi)^{3}} \frac{e^{\alpha\left(q^{* 2}-r^{2}\right)}}{q^{* 2}-r^{2}} .
$$

With this expression for $c^{P}\left(q^{* 2}\right)$, the quantization condition in eq. (54) is manifestly equivalent to that of ref. [7]. ${ }^{8}$

The equivalence can be extended straightforwardly to higher partial waves. The key point is that the vectors $\vec{k}^{*}$ and $\vec{r}$ are equal at the pole, as established above. Thus one can replace angular dependence on $\vec{k}^{*}$ with the same dependence on $\vec{r}$, with the difference being non-singular. The argument goes as follows (assuming $l>0$ ):

$$
\begin{aligned}
& c_{l m}^{P}\left(q^{* 2}\right)=\frac{1}{L^{3}} \sum_{\vec{k}} \frac{\omega_{k}^{*}}{\omega_{k}} \frac{e^{\alpha\left(q^{* 2}-k^{* 2}\right)}}{q^{* 2}-k^{* 2}} k^{* l} \sqrt{4 \pi} Y_{l m}\left(\theta^{*}, \phi^{*}\right) \\
& =\frac{1}{L^{3}} \sum_{\vec{k}} \frac{\omega_{k}^{*}}{\omega_{k}} \frac{e^{\alpha\left(q^{* 2}-k^{* 2}\right)}}{q^{* 2}-k^{* 2}} k^{* l} \sqrt{4 \pi} Y_{l m}\left(\theta^{*}, \phi^{*}\right) \\
& -\mathcal{P} \int \frac{d^{3} k}{(2 \pi)^{3}} \frac{\omega_{k}^{*}}{\omega_{k}} \frac{e^{\alpha\left(q^{* 2}-k^{* 2}\right)}}{q^{* 2}-k^{* 2}} k^{* l} \sqrt{4 \pi} Y_{l m}\left(\theta^{*}, \phi^{*}\right) \\
& =\frac{1}{\gamma L^{3}} \sum_{\vec{k}}\left\{\frac{e^{\alpha\left(q^{* 2}-r^{2}\right)}}{q^{* 2}-r^{2}} r^{* l} \sqrt{4 \pi} Y_{l m}\left(\theta_{r}, \phi_{r}\right)+g_{l m}(\vec{k})\right\} \\
& -\frac{1}{\gamma} \mathcal{P} \int \frac{d^{3} k}{(2 \pi)^{3}}\left\{\frac{e^{\alpha\left(q^{* 2}-r^{2}\right)}}{q^{* 2}-r^{2}} r^{* l} \sqrt{4 \pi} Y_{l m}\left(\theta_{r}, \phi_{r}\right)+g_{l m}(\vec{k})\right\} \\
& =\frac{1}{\gamma L^{3}} \sum_{\vec{k}} \frac{e^{\alpha\left(q^{* 2}-r^{2}\right)}}{q^{* 2}-r^{2}} r^{* l} \sqrt{4 \pi} Y_{l m}\left(\theta_{r}, \phi_{r}\right) \text {. }
\end{aligned}
$$

In the first step we have added an integral that vanishes, but is nevertheless useful for subsequent manipulations. In the next step we have rewritten the expression in terms of $\vec{r}$ [using spherical components $\left(r, \theta_{r}, \phi_{r}\right)$ ], leading to the presence of the non-singular function $g_{l m}(\vec{k})$. To make the final step, we note that the sum and integral over $g_{l m}$ cancel, and that the remaining integral over $\vec{k}$, which can be rewritten as an integral over $\vec{r}$, vanishes by rotational symmetry. The result is an expression given in ref. [7], although regularized differently. The precise relation is

$$
c_{l m}^{P}\left(q^{* 2}\right)=-\frac{\sqrt{4 \pi}}{\gamma L^{3}}\left(\frac{2 \pi}{L}\right)^{l-2} Z_{l m}^{d}\left[1 ;\left(q^{*} L / 2 \pi\right)^{2}\right]
$$

where as usual the equality holds up to terms which vanish exponentially with the box size. As already noted in sec. 3.2.2, with this relation, our general quantization condition eq. (56) agrees with that obtained in ref. [7].

${ }^{8}$ We use a different regularization, but the result should be independent of the ultraviolet regulator, and we have checked that our result agrees numerically with that obtained using the analytic regularization of ref. [7], up to terms which vanish exponentially as $L \rightarrow \infty$. 


\section{Quantization Condition in Perturbation Theory}

In this section we test the validity of the quantization condition in eq. (154) in perturbation theory. We assume an interaction potential $\lambda \pi^{4} / 4$ ! and work to linear order in $\lambda$. Specifically, we take the expressions for the s-wave phase-shift and two pion energy evaluated at $O(\lambda)$ and verify that they satisfy eq. (54).

The $\pi \pi$ phase-shift at $O(\lambda)$ is readily calculable and is given by

$$
\delta\left(q^{*}\right)=-\frac{\lambda q^{*}}{16 \pi E^{*}} .
$$

We stress that, for this exercise, we are considering indistiguishable "pions" without an isospin degree of freedom.

We now sketch the derivation of the two-pion energy at one-loop order and then in sec.5.2 check that they satisfy the quantization condition.

\subsection{Evaluation of the Two-Pion Energy in Perturbation Theory}

For the perturbative evaluation of the energy eigenvalues in a finite volume, we follow the steps in the derivation presented in section 4 of ref. [8], generalised to an arbitrary frame. We refer the reader to ref. [8] for a more complete description of the calculation. The energy eigenvalues are obtained by evaluating the time dependence of the Euclidean correlation function: ${ }^{9}$

$$
C_{\vec{q}_{1} \vec{q}_{2}}(t)=\left\langle 0\left|\pi_{-\vec{q}_{1}}(t) \pi_{-\vec{q}_{2}}(t) \pi(0)^{2}\right| 0\right\rangle,
$$

where

$$
\pi_{\vec{q}}(t)=\int d^{3} x \pi(\vec{x}, t) e^{i \vec{q} \cdot \vec{x}}
$$

is the Fourier transform of the pion field, which creates a pion with momentum $\vec{q}$ and destroys a pion with momentum $-\vec{q}$. The momenta $\overrightarrow{q_{i}}$ must take one of the values allowed by the box size, but are otherwise completely general. In particular, $\vec{P}=\vec{q}_{1}+\vec{q}_{2}$ need not vanish.

The two-pion energy can be deduced from the exponential dependence of the correlation function on $t$. Without loss of generality, we take $t>0$. At tree level $C_{\vec{q}_{1} \vec{q}_{2}}(t)$ can be represented by the diagram in fig. 3 (a), and one obtains

$$
C_{\vec{q}_{1} \vec{q}_{2}}^{(a)}(t)=2 \frac{e^{-\omega_{1} t}}{2 \omega_{1}} \frac{e^{-\omega_{2} t}}{2 \omega_{2}}
$$

where $\omega_{i}^{2}=\vec{q}_{i}^{2}+m^{2}$, and the factor of 2 arises since there are two possible contractions. The two-pion energy is thus $\omega_{1}+\omega_{2}$.

\footnotetext{
${ }^{9}$ In ref. [8] the discussion considered the $K \rightarrow \pi \pi$ amplitude, but for the calculation of two pion energies we do not need the initial kaon.
} 


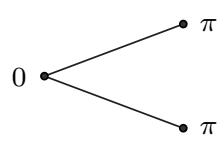

(a)

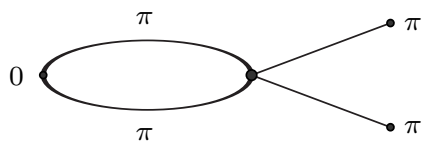

(b)

Figure 3: Contributions to the correlation function defined in eq. (76)): (a) tree-level diagram; (b) one-loop diagram giving the energy shift at $O(\lambda)$.

At one-loop order, only the diagram in fig. 3(b) gives rise to a shift in the two-pion energy. Following ref. [8], the contribution linear in $t$ is seen to be

$$
C_{\vec{q}_{1} \vec{q}_{2}}^{(b)}(t)=\sum_{\substack{\vec{q}_{1}^{\prime}, \vec{q}_{2}^{\prime} \\ \text { on }- \text { shell }}} \sum_{t^{\prime}=0}^{t}-\frac{\lambda}{L^{3}} \frac{e^{-\omega_{1}^{\prime} t^{\prime}}}{2 \omega_{1}^{\prime}} \frac{e^{-\omega_{2}^{\prime} t^{\prime}}}{2 \omega_{2}^{\prime}} \frac{e^{-\omega_{1}\left(t-t^{\prime}\right)}}{2 \omega_{1}} \frac{e^{-\omega_{2}\left(t-t^{\prime}\right)}}{2 \omega_{2}} .
$$

Here $\vec{q}_{1}^{\prime}$ and $\vec{q}_{2}^{\prime}$ are the loop momenta (with $\omega_{i}^{\prime}$ the corresponding energies), and the sum over $\vec{q}_{i}^{\prime}$ is over all "on-shell" intermediate states. These are the choices satisfying $\vec{q}_{1}^{\prime}+\vec{q}_{2}^{\prime}=\vec{P}$ and $\omega_{1}^{\prime}+\omega_{2}^{\prime}=\omega_{1}+\omega_{2}$. The number of such choices is denoted $\nu$. In the CM frame, this constraint implies $\omega_{1}^{\prime}=\omega_{2}^{\prime}=\omega_{1}=\omega_{2}$, so that all contributions are related by rotations, and all come with the same kinematical factor. For $\vec{P} \neq 0$, however, the on-shell constraint can be satisfied in two ways: (i) those related by a lab frame symmetry transformation, for which $\omega_{1}^{\prime}=\omega_{1}$ or $\omega_{2}^{\prime}=\omega_{2}$; and (ii) accidental choices of $\vec{q}_{1}^{\prime}$ not related by a lab frame symmetry transformation, for which $\omega_{1}^{\prime}$ equals neither $\omega_{1}$ or $\omega_{2}$, although $\omega_{1}^{\prime}+\omega_{2}^{\prime}=\omega_{1}+\omega_{2}$. The latter are absent in general, since they require special values of the pion masses. ${ }^{10}$ They are also absent for the simplest choices of momenta, e.g. $\vec{q}_{1}=(1,0,0)$ and $\vec{q}_{2}=(0,0,0)$ (in units of $\left.2 \pi / L\right)$, for which $\nu=2$ for all pion masses.

In the following, we assume that there are no accidental on-shell configurations. In this case, the kinematical factor is common to all terms in the sum over $t^{\prime}$ and one finds that the term linear in $t$ in $C^{(b)}$ is

$$
C_{\overrightarrow{q_{1}} \vec{q}_{2}}^{(b)}(t)=-t \nu \frac{\lambda}{L^{3}} \frac{e^{-\left(\omega_{1}+\omega_{2}\right) t}}{\left(4 \omega_{1} \omega_{2}\right)^{2}} .
$$

Combining this with the result for $C^{(a)}$ one finds, the energy shift $\Delta E=E-\omega_{1}-\omega_{2}$ to be

$$
\Delta E=\frac{1}{L^{3}} \frac{\lambda \nu}{8 \omega_{1} \omega_{2}} .
$$

Note that this holds for any allowed choice of $\omega_{i}$.

\subsection{Verification of the Quantization Condition}

We recall that the quantization condition is $4 \pi \tan (\delta) / q^{*}=1 / c^{P}\left(q^{* 2}\right)$, since we only have s-wave scattering. Verifying this turns out to be simpler if one uses the form of

\footnotetext{
${ }^{10}$ One example is $\vec{q}_{1}=(2,4,0), \vec{q}_{2}=(-2,1,0), \vec{q}_{1}^{\prime}=(1,5,0)$ and $\vec{q}_{2}^{\prime}=(-1,0,0)$ with $m^{2}=209 / 16$ (all quantities in units of $2 \pi / L)$.
} 
$c^{P}\left(q^{* 2}\right)$ given in eq. (69) , i.e. the form obtained in ref. [7]. Schematically this form is $c^{P}=\gamma^{-1} L^{-3} \sum_{\vec{k}}\left(q^{* 2}-r^{2}\right)^{-1}$. At leading order this condition is satisfied almost trivially. The allowed energies are those of two free pions each with momentum consistent with the boundary conditions. For any such choice we have $q^{* 2}=k^{* 2}=r^{2}$, as discussed in sec. 4 . Thus $c_{P}$ is infinite, requiring $\tan \delta=0$, which is consistent with the leading order result that $\delta=0$.

At next order we consider energies lying close to one of free pion values: $E=\omega_{1}+\omega_{2}+\Delta E$ where $\omega_{i}=\sqrt{\vec{q}_{i}{ }^{2}+m^{2}}$, with $\vec{q}_{i}$ being the allowed finite-volume momenta. The energy shift $\Delta E$ is of $O(\lambda)$. Thus in each of the $\nu$ terms in the summation which at leading order had $q^{* 2}=r^{2}$ the denominators $q^{* 2}-r^{2}$ are now of $O(\lambda)$, and hence $c_{P}$ is of $O(1 / \lambda)$. All other terms in the summation, as well as the principal part integral required for regularization, are of $O(1)$ and thus subleading. Keeping only the leading terms, the quantization condition becomes

$$
\delta=\left.\frac{q^{*}}{4 \pi} \frac{\gamma L^{3}}{\nu} \Delta E \frac{d\left(q^{* 2}-r^{2}\right)}{d E}\right|_{q^{* 2}=r^{2}} .
$$

Here we have made use of the result, to be established below, that all $\nu$ relevant terms give the same contribution to $c^{P}$.

The first part of the derivative is readily evaluated. Recalling that $4\left(q^{* 2}+m^{2}\right)=E^{* 2}=$ $E^{2}-P^{2}$, and noting that the derivative is to be performed at fixed $P$ and $m$, we have

$$
\frac{d q^{* 2}}{d E}=\frac{E}{2}
$$

Next, using the definition (61) for $\vec{r}$, and noting the $\vec{k}$ is held fixed, we have

$$
\frac{d r^{2}}{d E}=\frac{d r_{\|}^{2}}{d E}=\left(k_{\|}-P / 2\right)^{2} \frac{d \gamma^{-2}}{d E}=\left(k_{\|}-P / 2\right)^{2} \frac{2 P^{2}}{E^{3}} .
$$

Combining (83) and (84) we find

$$
\frac{d\left(q^{* 2}-r^{2}\right)}{d E}=\frac{E^{4}-\left(2 k_{\|}-P\right)^{2} P^{2}}{2 E^{3}} .
$$

This expression simplifies when evaluated for two on-shell pions (i.e. for $q^{* 2}=r^{2}$ ). The four-vectors of the two pions in the lab frame are then

$$
q_{1}^{\mu}=\left(\omega_{1}, \vec{k}\right), \quad q_{2}^{\mu}=\left(\omega_{2}, \vec{P}-\vec{k}\right) .
$$

Observing that

$$
0=\left(q_{1}+q_{2}\right)^{\mu}\left(q_{1}-q_{2}\right)_{\mu}=E\left(\omega_{1}-\omega_{2}\right)-\left(2 k_{\|}-P\right) P
$$

the derivative at the pole is readily seen to be

$$
\left.\frac{d\left(q^{* 2}-r^{2}\right)}{d E}\right|_{q^{* 2}=r^{2}}=\frac{E^{2}-\left(\omega_{1}-\omega_{2}\right)^{2}}{2 E}=\frac{2 \omega_{1} \omega_{2}}{E} .
$$


Note that this result is the same for all $\nu$ contributions to the sum in $c^{P}$.

Inserting this result in the quantization condition and rearranging gives

$$
\delta=\frac{q^{*}}{16 \pi E^{*}} \frac{\Delta E L^{3}}{\nu} 8 \omega_{1} \omega_{2}
$$

This is consistent with the results for $\delta$ and $\Delta E$ given respectively in eqs. (75) and (81) above, confirming the validity of the quantization condition at $O(\lambda)$ in perturbation theory.

\section{Finite-Volume Effects in Matrix Elements}

In this section we generalise the Lellouch-Lüscher formula [5] for the finite-volume effects in $K \rightarrow \pi \pi$ matrix elements from the centre-of-mass frame to a moving frame in which the two-pions have total momentum $\vec{P}$. We find the following relation between the matrix elements of the effective Weak Hamiltonian density, $\mathcal{H}_{W}$, in infinite and finite volumes:

$$
\left|A_{W}\right|^{2}=8 \pi V^{2} \frac{m_{K} E^{2}}{q^{* 2}}\left\{\delta^{\prime}\left(q^{*}\right)+\phi^{P^{\prime}}\left(q^{*}\right)\right\}\left|M_{W}\right|^{2}
$$

where the ' represents the derivative with respect to $q^{*}, \delta$ is the s-wave phase shift, $\phi^{P}$ the kinematic function defined in eq. (154), $V=L^{3}$ is the spatial volume, and the $K \rightarrow \pi \pi$ matrix elements in infinite and finite volumes are respectively

$$
A_{W}={ }_{\infty}\left\langle\pi \pi ; E, \vec{P}\left|\mathcal{H}_{W}(0)\right| K ; \vec{P}\right\rangle_{\infty}
$$

and

$$
M_{W}={ }_{V}\left\langle\pi \pi ; E, \vec{P}\left|\mathcal{H}_{W}(0)\right| K ; \vec{P}\right\rangle_{V}
$$

(as indicated by the subscripts). The infinite volume states have the usual relativistic normalizations, while the finite volume states are normalized to unity. Note that for this formula we are assuming that $\mathcal{H}_{W}(0)$ does not insert energy or momentum. In particular, for both infinite and finite volume the energy of the two-pion state equals that of the initial kaon, $E^{2}=m_{K}^{2}+P^{2}$. We comment on the generalisation below.

The result (90) is valid, as usual, up to corrections vanishing exponentially with $L$. It also assumes that one can neglect the scattering amplitude in the $l=2,4, \ldots$ waves at $E^{*}=m_{K}$. Note that this is a stronger assumption for $\vec{P} \neq 0$ than for $\vec{P}=0$. In the latter case, the first higher partial wave which contributes has $l=4$, due to the cubic symmetry. For a moving frame, however, the symmetry is reduced and the leading contribution comes from $l=2$. Technically this arises because $c_{20}^{P} \propto Z_{20}^{d} \neq 0$, as shown explicitly in ref. [7].

In the centre-of-mass frame the original derivation of the formula in eq. (90) proceeded by using degenerate perturbation theory [5], in which the volume was chosen so that, in the absence of weak interactions, the kaon and the two-pion states are degenerate. This degeneracy is then broken by the weak interactions. In the derivation of ref. [6] the factor relating the infinite and finite volume matrix elements was interpreted in terms of the 


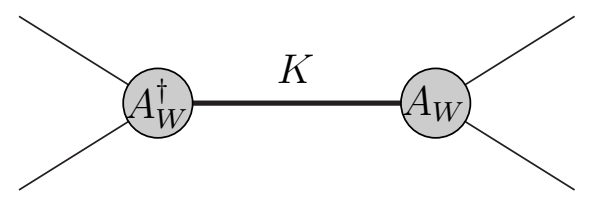

Figure 4: $O(\lambda)$ contribution to the $\pi \pi$ scattering amplitude (see text). The thick line represents the propagator of a kaon, which is off-shell by an amount of $O(\lambda) . A_{W}$ represents the infinite-volume $K \rightarrow \pi \pi$ matrix element.

density of states of the two-pion system. We now derive eq. (90) using both approaches in turn. For simplicity, we continue to treat the pions as identical, ignoring the isospin degree of freedom. The final result applies, however, separately for each isospin state (using the corresponding phase shift).

\subsection{Derivation using degenerate perturbation theory}

In this section we generalise the derivation of ref. [5] for the finite-volume effects in matrix elements to a moving frame. We start by considering the case with no weak interactions (so that $K \rightarrow \pi \pi$ decays are not possible) and take the volume such that the energy of a kaon with momentum $\vec{P}$ is equal to the energy of one of the finite-volume two-pion states with the same total momentum. ${ }^{11}$ The weak interactions are now introduced as an (arbitrarily weak) perturbation which breaks the degeneracy between the kaon and two-pion states. The energy eigenvalues are then

$$
E=E_{0} \pm \lambda V\left|M_{W}\right|
$$

where $E_{0}^{2}=P^{2}+m_{K}^{2}$ and $\lambda$ is a parameter introduced to facilitate the counting of orders of perturbation theory and is set to 1 in the final results. The factor of $V$ is present because in eq. (92) we have defined $M_{W}$ to be the matrix element of the Hamiltonian density.

We can also calculate the shifts in (93) by taking into account the effect of the weak interactions on the phase shift, which then impacts the finite-volume energies through the quantization condition. The only contribution to the scattering amplitude (and thus phase shift) linear in $\lambda$ is that from the diagram shown in fig.4] [5]. The energy and momentum of the external $\pi \pi$ states are chosen to be $(E, \vec{P})$, where $E$ is one of the finite volume energies in eq. (93)). The kaon propagator is thus off shell by $O(\lambda)$, and when combined with the two $K \pi \pi$ vertices, each of $O(\lambda)$, the result is a contribution to the s-wave scattering amplitude of first order in perturbation theory:

$$
\Delta M_{s}=\mp \lambda \frac{\left|A_{W}\right|^{2}}{2 E_{0} V\left|M_{W}\right|} e^{2 i \delta\left(q^{*}\right)},
$$

\footnotetext{
${ }^{11} \mathrm{We}$ are considering here only the two-pion states with an s-wave component, so that their energy is determined by the quantization condition eq. (54) using the s-wave scattering amplitude. These states go over to pure s-wave states as the volume is sent to infinity.
} 
where $\delta$ represents the unperturbed phase-shift. Using eq. (52) we obtain the expression for $\bar{\delta}$, the perturbed phase-shift (i.e. the phase-shift including $O(\lambda)$ terms):

$$
\bar{\delta}\left(q^{*}\right)=\delta\left(q^{*}\right) \mp \frac{\lambda}{32 \pi} \frac{q^{*}\left|A_{W}\right|^{2}}{m_{K} E_{0} V\left|M_{W}\right|} .
$$

In order to obtain eq. (90) we

1. use the quantization condition to replace $\bar{\delta}\left(q^{*}\right)$ by $n \pi-\phi^{P}\left(q^{*}\right)$;

2. expand $\phi^{P}\left(q^{*}\right)$ and $\delta\left(q^{*}\right)$ around the unperturbed point $q_{0}^{*}$, where $E_{0}^{* 2}=m_{K}^{2}=$ $4 q_{0}^{* 2}+m^{2}$

$$
\phi^{P}\left(q^{*}\right) \simeq \phi^{P}\left(q_{0}^{*}\right)+\Delta q^{*} \phi^{P \prime}\left(q_{0}^{*}\right) \quad \text { and } \quad \delta\left(q^{*}\right) \simeq \delta\left(q_{0}^{*}\right)+\Delta q^{*} \delta^{\prime}\left(q_{0}^{*}\right)
$$

with

$$
\Delta q^{*}= \pm \frac{\lambda E}{4 q^{*}} V\left|M_{W}\right|
$$

3. Note that $\phi^{P}\left(q_{0}^{*}\right)=n \pi-\delta\left(q_{0}^{*}\right)$ and equate the $O(\lambda)$ terms on both sides of eq. (95).

\subsection{Derivation of eq. (90) using the density of states}

In this section we derive eq. (90) by generalising the arguments of ref. [6]. Let $\sigma(\vec{x}, t)$ be an interpolating operator which can create two-pion states from the vacuum and consider the two point correlation function in finite volume:

$$
\begin{aligned}
C_{\sigma}^{V}(t) & \equiv \int d^{3} x e^{i \vec{P} \cdot \vec{x}}\langle 0|\sigma(\vec{x}, t) \sigma(\overrightarrow{0}, 0)| 0\rangle_{V} \\
& =V \sum_{n}|\langle 0|\sigma(0)| \pi \pi ; \vec{P}, n\rangle|^{2} e^{-E_{n} t} \\
& \underset{V \rightarrow \infty}{\longrightarrow} V \int d E \rho_{V}(E)\left|\langle 0|\sigma(\overrightarrow{0}, 0)| \pi \pi ; E, \vec{P}\rangle_{V}\right|^{2} e^{-E t}
\end{aligned}
$$

In eq. (100) $\rho_{V}(E)$ is the density of states and can be evaluated using the quantization condition in eq. (54). This condition can be rewritten as

$$
\delta\left(q^{*}\right)+\phi^{P}\left(q^{*}\right)=n \pi
$$

with $n$ an integer. We argue below that, for large enough $L, \delta+\phi^{P}$ increases monotonically, so that $n$ counts the number of states. Given this, the density of states is given by

$$
\rho_{V}(E)=\frac{d n}{d E}=\frac{E}{4 \pi q^{*}}\left\{\delta^{\prime}\left(q^{*}\right)+\phi^{P \prime}\left(q^{*}\right)\right\} .
$$

The monotonicity of $\delta+\phi^{P}$ follows because, as explained below, $\phi^{P}$ is a monotonically increasing function of $q^{*}$ whose derivative is proportional to $L$. By contrast the dependence of $\delta$ on $q^{*}$ is volume independent. Thus, although $\delta$ need not be an increasing function of $q^{*}$, its dependence will be overwhelmed by that of $\phi^{P}$ for large enough $L$. 
The behaviour of $\phi^{P}$ can be seen most easily using the form obtained in ref. [7]:

$$
\phi^{P}\left(q^{*}\right)=\tan ^{-1}\left[q^{*} /\left(4 \pi c^{P}\right)\right], \quad c^{P}=\left(\gamma L^{3}\right)^{-1} \sum_{\vec{k}}\left(q^{* 2}-r^{2}\right)^{-1} .
$$

Aside from the factor of $\gamma^{-1}, c^{P}$ decreases monotonically between the poles which occur whenever there is a free two-pion state in volume $L^{3}$ with total momentum $\vec{P}$. As $L$ increases, the spacing in $q^{*}$ between such states decreases, so the derivative $c^{P \prime}$ becomes more negative, and overwhelms any positive contribution to the derivative from $\gamma^{-1}$. This behaviour of $c^{P}$ implies that $\phi^{P}$ increases monotonically at large enough $q^{\star}$. Furthermore, since $\phi^{P}$ increases by $\pi$ for each transit between poles, whose spacing $\sim 1 / L$, the derivative $\phi^{P \prime}$ is proportional to $L$.

We now return to the main line of the argument. The infinite-volume correlation function corresponding to $C_{\sigma}^{V}$ is given by

$$
C_{\sigma}^{\infty}(t)=\frac{1}{16 \pi^{2}} \int d E \frac{q^{*}}{E^{*}}\left|\langle 0|\sigma(\overrightarrow{0}, 0)| \pi \pi ; \vec{P}, E\rangle_{\infty}\right|^{2} e^{-E t}
$$

where $E^{* 2}=4 q^{* 2}+m^{2}=E^{2}-P^{2}$ as above. Comparing eqs. (100) and (104) we obtain the correspondence between the infinite-volume and finite-volume kets:

$$
|\pi \pi ; \vec{P}, E\rangle_{\infty} \Leftrightarrow 4 \pi \sqrt{\frac{V E^{*} \rho_{V}\left(q^{*}\right)}{q^{*}}}|\pi \pi ; \vec{P}, E\rangle_{V}
$$

Similarly, by considering correlation functions of interpolating operators for single particle states, we find:

$$
|K ; \vec{P}\rangle_{\infty} \Leftrightarrow \sqrt{2 E V}|K ; \vec{P}\rangle_{V}
$$

with $E^{2}=P^{2}+m_{K}^{2}$. Eq. (106) is simply the relation between the wavefunctions of states normalized relativistically ( $2 E$ particles per unit volume) and non-relativistically in a finite-volume (1 particle in volume $V$ ).

Combining eqs. (105) and (106) we obtain the required relation between the matrix elements in infinite and finite-volume in eq.(90) .

In eq. (90) the matrix elements correspond to physical processes and energy and momentum are conserved. However, eqs. (105) and (106) can be applied more generally. One such application is to the chiral extrapolation of the matrix elements computed in lattice simulations. Since these are currently performed at unphysically large masses for the $u$ and $d$ quarks, the results have to be extrapolated to the physical point. Chiral perturbation theory $(\chi \mathrm{PT})$ can be useful in guiding this extrapolation. For example, in ref. [8] it was proposed to fix the low-energy constants which appear in $\chi \mathrm{PT}$ at next-to-leading order by computing $K \rightarrow \pi \pi$ matrix elements with the kaon and one of the final state pions at rest and varying the momentum of the second pion. Equations (105) and (106), with different energies and momenta for the kaon and two-pion system, can be combined to determine the finite-volume effects also in such cases. 


\section{$7 \quad$ Summary and Conclusions}

In this paper, we have provided a field theoretic derivation of the finite volume energy shift for two hadron states in a moving frame, confirming the result obtained by ref. [7] using a relativistic quantum mechanical approach. We have also determined the finite-volume corrections in matrix elements of local composite operators with an initial and/or final state consisting of two hadrons, thus generalising the Lellouch-Lüscher factor to moving frames. The path is therefore now open to numerical studies of two pion energies and matrix elements in a moving frame. As described in the introduction, there are a number of important applications which depend upon, or are greatly simplified by, working with $\vec{P} \neq 0$ as well as $\vec{P}=0$.

Our work provides three new results. The first is technical: we give a simple derivation of the summation formulae needed to determine finite-volume effects. It has the advantages of conceptual simplicity, and that it can be readily applied in any frame. In addition, it provides a straightforward way of calculating the required regularized sums numerically. It is of comparable numerical efficiency to the method used in refs. [3] and [7], based on analytic regularization.

Our second new result is a method to determine the energies of finite volume states, to all orders in perturbation theory in $1 / L$, based entirely in field theory. This is in contrast to the method developed in refs. [2,3] for the centre-of-mass frame. There it is shown that, in the centre-of-mass frame, the two-particle energies in a general relativistic field theory are related to those of an auxiliary non-relativistic quantum mechanical (NRQM) system in the same finite volume, with an energy-dependent potential chosen to reproduce the relativistic scattering phase shifts. The analysis is then carried out in the NRQM system. Perturbation theory in $1 / L$ is developed in ref. [2], with ref. [3] providing the generalisation to all orders in $1 / L$. The use of a NRQM theory is a technical device, and does not require the underlying two-particle system to be non-relativistic. It reflects the fact that the dominant finite volume effects come from nearly on-shell two-particle intermediate states. Nevertheless, we believe that it is conceptually simpler to avoid the need for an auxiliary NRQM theory, and this is what our approach allows. In addition, we obtain the general result with multiple non-vanishing partial wave amplitudes in a very straightforward fashion.

The particular advantage of our approach, however, is that it is readily generalisable to a moving frame. The general formalism remains unchanged, but the required kinematical functions, which arise from the relation between two-particle loop momentum sums and integrals, depend on $\vec{P}$. The generalisation of the method using the auxiliary NRQM theory is less obvious, in part because of the mismatch between relativistic and nonrelativistic energies for $\vec{P} \neq 0$. This is finessed in ref. [7] by the use of a relativistic two particle wave function, satisfying the Klein-Gordon equation. The wavefunction is subject to periodic boundary conditions in the moving frame, and the key step is to convert these to boundary conditions in centre-of-mass coordinates. One would expect the latter to involve unequal times because of the Lorentz boost, but the authors of ref. [7] argue that this is not the case. Their argument is based on the observation that outside of the interaction region the wave function is that of two free particles and can readily be 
shown to be independent of the relative time in the centre-of-mass frame. From this they conclude that the boundary conditions can be applied at equal times also in the centre-ofmass frame, with the effect of the boost causing an elongation of the box in the direction of $\vec{P}$. We were left with the question as to whether this procedure correctly corresponds to periodic boundary conditions in the moving frame (including inside the interaction region) and this question partially motivated us to develop the alternative approach presented here. Since our final results for the quantization condition can be brought into the same form as those of ref. [7], we have indirectly confirmed their assumptions.

As a check of our result (and thus also that of ref. [7]) we have calculated the two-pion energies to leading non-trivial order in the perturbative expansion in a $\lambda \phi^{4}$ theory, and confirmed the quantization formula in a general moving frame.

Our final new result is the generalization to moving frames of the Lellouch-Lüscher factor which contains the finite-volume effects in $K \rightarrow \pi \pi$ matrix elements. This turns out to require only a simple kinematical change: the non-trivial effects of working in a moving frame are already incorporated in the functions $c_{l m}^{P}$ that determine the two-particle energies in finite volumes.

\section{Acknowledgements}

We thank Norman Christ and Takeshi Yamazaki for communicating their work to us prior to its release. We thank them, and also Maarten Golterman, Kari Rummukainen and Massimo Testa for helpful discussions and correspondence. CTS thanks the Institute for Nuclear Theory for its hospitality during the Programme on Effective Field Theories, QCD and Heavy Hadrons and the Department of Energy for partial support during the later stages of this work. SRS thanks the University of Southampton for hospitality during the initial part of this work and acknowledges support from a PPARC Visiting Fellowship (PPA/V/S/2003/00006). We also acknowledge support from PPARC grants PPA/G/O/2002/00468 and PPA/G/S/2003/00093 and from DOE grant DE-FG0296ER40956.

\section{References}

[1] M. Luscher, Commun. Math. Phys. 104 (1986) 177.

[2] M. Luscher, Commun. Math. Phys. 105 (1986) 153.

[3] M. Luscher, Nucl. Phys. B 354 (1991) 531.

[4] M. Luscher, Nucl. Phys. B 364 (1991) 237.

[5] L. Lellouch and M. Luscher, Commun. Math. Phys. 219, 31 (2001) arXiv:hep-lat/0003023.

[6] C. J. D. Lin, G. Martinelli, C. T. Sachrajda and M. Testa, Nucl. Phys. B 619 (2001) 467 arXiv:hep-lat/0104006. 
[7] K. Rummukainen and S. A. Gottlieb, Nucl. Phys. B 450 (1995) 397 arXiv:hep-lat/9503028.

[8] C. J. D. Lin, G. Martinelli, E. Pallante, C. T. Sachrajda and G. Villadoro, Nucl. Phys. B 650 (2003) 301 arXiv:hep-lat/0208007.

[9] C. h. Kim and N. H. Christ, Nucl. Phys. Proc. Suppl. 119 (2003) 365 arXiv:hep-lat/0210003;

C. Kim, Nucl. Phys. Proc. Suppl. 129 (2004) 197 arXiv:hep-lat/0311003.

[10] P. F. Bedaque, Phys. Lett. B 593, 82 (2004) arXiv:nucl-th/0402051;

G. M. de Divitiis, R. Petronzio and N. Tantalo, Phys. Lett. B 595, 408 (2004) arXiv:hep-lat/0405002;

G. M. de Divitiis and N. Tantalo, arXiv:hep-lat/0409154,

C. T. Sachrajda and G. Villadoro, Phys. Lett. B 609 (2005) 73 arXiv:hep-lat/0411033;

P. F. Bedaque and J. W. Chen, Phys. Lett. B 616, 208 (2005) arXiv:hep-lat/0412023;

B. C. Tiburzi, arXiv:hep-lat/0504002.

J. M. Flynn, A. Juttner and C. T. Sachrajda, arXiv:hep-lat/0506016.

[11] N.H. Christ, C. Kim and T. Yamazaki, "Finite Volume Corrections to the TwoParticle Decay of States with Non-Zero Momentum".

[12] S. R. Beane, P. F. Bedaque, A. Parreno and M. J. Savage, Phys. Lett. B 585, 106 (2004) arXiv:hep-lat/0312004. 